\title{
THE CARDINALITY OF REDUCED POWER SET ALGEBRAS
}

\author{
ALAN D. TAYLOR
}

(Communicated by Thomas J. Jech)

\begin{abstract}
We prove a general result on the cardinality of reduced powers of structures via filters that has several consequences including the following: if $I$ is a uniform, countably complete ideal on the real line $\mathscr{R}$ and $\mathscr{B}$ is the Boolean algebra of subsets of $\mathscr{R}$ modulo $I$, then $|\mathscr{B}|>2^{\aleph_{0}}$ and if $2^{\nu} \leq 2^{\aleph_{0}}$ for all $\nu<2^{\aleph_{0}}$ then $|\mathscr{B}|=2^{2^{\aleph_{0}}}$. This strengthens some results of Kunen and Pelc [7] and Prikry [8] obtained by Boolean ultrapower techniques. Our arguments are all combinatorial and some applications are included.
\end{abstract}

1. Introduction. Our notation and terminology are fairly standard. If $A$ and $B$ are sets then ${ }^{A} B$ denotes the set of all functions mapping $A$ into $B$ and $\mathscr{P}(A)$ denotes the power set of $A$. Throughout, $\kappa$ and $\lambda$ will denote infinite cardinals. An ideal $I$ on $\kappa$ is a collection of subsets of $\kappa$ that is closed under subset formation and finite unions. All the ideals we will consider are assumed to be proper $(I \neq \mathscr{P}(\kappa))$ but not necessarily $\kappa$-complete (i.e. closed under unions of size less than $\kappa$ ) or even uniform (i.e. containing all subsets of $\kappa$ of size less than $\kappa$ ). If $I$ is an ideal on $\kappa$ then $I^{*}$ denotes the filter on $\kappa$ that is dual to $I$; that is, $X \in I^{*}$ iff $\kappa-X \in I$, and $I^{+}$denotes $\mathscr{P}(\kappa)-I$.

If $I$ is an ideal on $\kappa$ and $B$ is an arbitrary set then the reduced power of $B$ (via $\left.I^{*}\right)$, denoted $\prod_{\kappa} B / I^{*}$, is the set of all equivalence classes of functions in ${ }^{\kappa} B$ where we identify two such functions $f$ and $g$ if $\{\alpha<\kappa: f(\alpha) \neq g(\alpha)\} \in I$. The Boolean algebra $\mathscr{P}(\kappa) / I$ is the set of all equivalence classes of subsets of $\kappa$ with the induced ordering from $\subseteq$, where we identify two such subsets $X$ and $Y$ if their symmetric difference $X \triangle Y$ is in $I$.

Since we will be comparing $\mathscr{P}(\kappa) / I$ and $\prod_{\kappa} \kappa / I^{*}$ it is worth pointing out the following (see [3 or 1, p. 338]). The function which associates to each $X \subseteq \kappa$ its characteristic function induces a natural bijection between $\mathscr{P}(\kappa) / I$ and $\prod_{\kappa} 2 / I^{*}$ where, of course, $2=\{0,1\}$. Hence $|\mathscr{P}(\kappa) / I|=\left|\prod_{\kappa} 2 / I^{*}\right| \leq\left|\prod_{\kappa} \kappa / I^{*}\right|$. In general, the inequality cannot be replaced by equality since trivially $\left|\prod_{\kappa} \kappa / I^{*}\right| \geq \kappa$ (consider the constant functions from $\kappa$ to $\kappa$ ) but $|\mathscr{P}(\kappa) / I|=2$ if $I$ is a prime (i.e. maximal) ideal on $\kappa$.

2. The main results. At the heart of our first theorem is the following trivial observation: there is a natural bijection between $\kappa$-sequences of subsets of $\lambda$ and $\lambda$ sequences of subsets of $\kappa$. This correspondence is given by $\left(X_{\alpha}: \alpha<\kappa\right) \leftrightarrow\left(Y_{\beta}: \beta<\right.$ $\lambda$ ) where $\alpha \in Y_{\beta}$ iff $\beta \in X_{\alpha}$. The proof of the following result consists of simply verifying that if $I$ is a $\lambda^{+}$-complete ideal on $\kappa$ then this correspondence induces a (well-defined) bijection between $\prod_{\kappa} \mathscr{P}(\lambda) / I^{*}$ and ${ }^{\lambda}(\mathscr{P}(\kappa) / I)$.

Received by the editors March 20, 1987.

1980 Mathematics Subject ('lassification (1985 Revision). Primary 03E05. Secondary 06E05. 
THEOREM 2.1. If I is a $\lambda^{+}$-complete ideal on $\kappa$ then

$$
\left|\prod_{\kappa}^{\lambda} 2 / I^{*}\right|=\left|\prod_{\kappa} 2 / I^{*}\right|^{\lambda} \text {. }
$$

ProOF. Define $\phi: \prod_{\kappa}{ }^{\lambda} 2 / I^{*} \rightarrow^{\lambda}\left(\prod_{\kappa} 2 / I^{*}\right)$ as follows: if $[f] \in \prod_{\kappa}{ }^{\lambda} 2 / I^{*}$, then $f: \kappa \rightarrow{ }^{\lambda} 2$ and we set

$$
\phi([f])=\left\langle\left[f_{\beta}\right]: \beta<\lambda\right\rangle
$$

where $f_{\beta}: \kappa \rightarrow 2$ is given by $f_{\beta}(\alpha)=f(\alpha)(\beta)$. Note that $\left[f_{\beta}\right] \in \prod_{\kappa} 2 / I^{*}$.

To complete the proof of the theorem it suffices to show that $\phi$ is a well-defined bijection.

Claim 1. $\phi$ is well defined.

ProOF. Suppose that $f, g: \kappa \rightarrow{ }^{\lambda} 2$ and $[f]=[g]$. Let $X=\{\alpha<\kappa: f(\alpha)=$ $g(\alpha)\}$. Then $X \in I^{*}$. Fix $\beta<\lambda$ and let $Y_{\beta}=\left\{\alpha<\kappa: f_{\beta}(\alpha)=g_{\beta}(\alpha)\right\}$. We claim that $X \subseteq Y_{\beta}$. That is, if $\alpha \in X$ then $f(\alpha)=g(\alpha)$ so $f(\alpha)(\beta)=g(\alpha)(\beta)$. But then $f_{\beta}(\alpha)=g_{\beta}(\alpha)$ so $\alpha \in Y_{\beta}$ as desired. But now since $X \in I^{*}$ and $X \subseteq Y_{\beta}$ we have $Y_{\beta} \in I^{*}$ and so $\left[f_{\beta}\right]=\left[g_{\beta}\right]$. Since $\beta$ was arbitrary this shows that $\left\langle\left[f_{\beta}\right]: \beta\langle\lambda\rangle=\right.$ $\left\langle\left[g_{\beta}\right]: \beta<\lambda\right\rangle$. Thus $\phi([f])=\phi([g])$ and so $\phi$ is well defined as claimed.

ClaIM. $\phi$ is one-to-one.

PROOF. Suppose that $f, g: \kappa \rightarrow{ }^{\lambda} 2$ and assume that $\phi([f])=\phi([g])$. Then for every $\beta<\lambda$ we have $\left[f_{\beta}\right]=\left[g_{\beta}\right]$ and so $Y_{\beta} \in I^{*}$ where $Y_{\beta}=\left\{\alpha<\kappa: f_{\beta}(\alpha)=\right.$ $\left.g_{\beta}(\alpha)\right\}$. Let $Y=\bigcap_{\beta<\lambda} Y_{\beta}$. Since $I$ is $\lambda^{+}$-complete we have $Y \in I^{*}$. Now let $X=\{\alpha<\kappa: f(\alpha)=g(\alpha)\}$. We claim that $Y \subseteq X$. That is, if $\alpha \in Y$ then for every $\beta<\lambda$ we have $\alpha \in Y_{\beta}$, and so $f_{\beta}(\alpha)=g_{\beta}(\alpha)$. But then for every $\beta<\lambda$ we have $f(\alpha)(\beta)=g(\alpha)(\beta)$ and so the functions $f(\alpha)$ and $g(\alpha)$ are equal. Hence $\alpha \in X$. Now, since $Y \in I^{*}$ and $Y \subseteq X$ we have $X \in I^{*}$ and so $[f]=[g]$ as desired.

Claim 3. $\phi$ is onto.

ProOF. Suppose $\left\langle\left[f_{\beta}\right]: \beta\langle\lambda\rangle \in{ }^{\lambda} \prod_{\kappa} 2 / I^{*}\right.$. Define $f: \kappa \rightarrow{ }^{\lambda} 2$ by $f(\alpha)(\beta)=$ $f_{\beta}(\alpha)$. Then $[f] \in \prod_{\kappa}{ }^{\lambda} 2 / I^{*}$ and clearly $\phi([f])=\left\langle\left[f_{\beta}\right]: \beta<\lambda\right\rangle$.

Specializing Theorem 2.1 to the case where $\kappa=2^{\lambda}$ and making use of some known results yields the following.

THEOREM 2.2. Suppose $I$ is a $\lambda^{+}$-complete ideal on $\kappa=2^{\lambda}$. Then

(i) $|\mathscr{P}(\kappa) / I|^{\lambda}=\left|\prod_{\kappa} \kappa / I^{*}\right| \geq \kappa$, and

(ii) if $I$ is also uniform (i.e. $[\kappa]^{<\kappa} \subseteq I$ ), then $|\mathscr{P}(\kappa) / I|>\kappa$.

ProOF. Part (i) follows immediately from Theorem 2.1 and our comments regarding $\mathscr{P}(\kappa) / I$ and $\prod_{\kappa} 2 / I^{*}$. For part (ii) we need the following well-known result (see [3, p. 204]): for every infinite cardinal $\gamma$ there exists a family $\mathscr{F} \subseteq{ }^{\gamma} \gamma$ with $|\mathscr{F}|=\gamma^{+}$and $|\{\alpha<\gamma: f(\alpha)=g(\alpha)\}|<\gamma$ whenever $f, g \in \mathscr{F}$ and $f \neq g$. An immediate consequence of this is the fact that if $I$ is a uniform ideal on $\kappa$ then $\left|\prod_{\kappa} \kappa / I^{*}\right| \geq \kappa^{+}$. To complete the proof of (ii), let $\mu=|\mathscr{P}(\kappa) / I|$ and suppose for contradiction that $\mu \leq \kappa$. Then

$$
\kappa^{+} \leq\left|\prod_{\kappa} \kappa / I^{*}\right|=|\mathscr{P}(\kappa) / I|^{\lambda}=\mu^{\lambda} \leq \kappa^{\lambda}=\left(2^{\lambda}\right)^{\lambda}=2^{\lambda}=\kappa .
$$

Finally, if we specialize still further and again use some known results we obtain the following. 
THEOREM 2.3. Suppose $I$ is an $\omega_{1}$-complete ideal on the real line $\mathscr{R}$. Then

(i) $|\mathscr{P}(\mathscr{R}) / I|=\left|\prod_{\mathscr{R}} \mathscr{R} / I^{*}\right|=\left|\prod_{\mathscr{R}} \mathscr{R} / I^{*}\right|^{\omega}$,

(ii) if $I$ is uniform then $|\mathscr{P}(\mathscr{R}) / I|>2^{\aleph_{0}}$, and

(iii) if $I$ is uniform and $2^{\nu} \leq 2^{\aleph_{0}}$ for all $\nu<2^{\aleph_{0}}$ then $|\mathscr{P}(\mathscr{R}) / I|=2^{2^{\aleph_{0}}}$.

Proof. Part (i) follows immediately from Theorem 2.2(i) and the following result of Comfort-Hager [2] and Monk-Sparks [6]: if $B$ is an infinite $\omega_{1}$-complete Boolean algebra, then $|B|^{\omega}=|B|$. Since $\mathscr{P}(\mathscr{R}) / I$ is $\omega_{1}$-complete and infinite, this gives us that $|\mathscr{P}(\mathscr{R}) / I|=|\mathscr{P}(\mathscr{R}) / I|^{\omega}$.

Part (ii) is an immediate consequence of Theorem 2.2(ii).

Part (iii) requires the following well-known result of Tarski (see [3, p. 204]): if $\gamma$ is an infinite cardinal and $2^{\gamma}=\gamma$ then there exists a family $\mathscr{F} \subseteq{ }^{\gamma} \gamma$ with $|\mathscr{F}|=2^{\gamma}$ and $|\{\alpha<\gamma: f(\alpha)=g(\alpha)\}|<\gamma$ whenever $f, g \in \mathscr{F}$ and $f \neq g$. (To see this, let $\left\langle X_{\beta}^{\alpha}: \beta<\gamma_{\alpha}\right\rangle$ be an enumeration of $\mathscr{P}(\alpha)$ in order-type $\gamma_{\alpha} \leq \gamma$ for each $\alpha<\gamma$ and for each $X \subseteq \gamma$ define $f_{X}: \gamma \rightarrow \gamma$ by $f_{X}(\alpha)=\beta$ iff $X \cap \alpha=X_{\beta}^{\alpha}$.) In the context of (iii), with $\gamma=2^{\aleph_{0}}(=|\mathscr{R}|)$, this gives us that $\left|\prod_{\mathscr{R}} \mathscr{R} / I^{*}\right| \geq 2^{2^{\aleph_{0}}}$, and so (iii) now follows from (i).

The second equality in part (i) of Theorem 2.3 should be compared with Keisler's result [5] that $\left|\prod \mathscr{R} / D\right|=|\Pi \mathscr{R} / D|^{\omega}$ for any countably incomplete ultrafilter $D$ on $\omega$. Part (ii) of Theorem 2.3 eliminates some saturation requirements from an old (unpublished) result of Kunen (recently rediscovered by Pelc [7]) and part (iii) does the same for a result of Prikry [8]. The proofs of Kunen, Pelc and Prikry all employed Boolean ultrapowers.

3. Some applications. We conclude with a few applications of the results in $\S 2$.

Application 1. We begin with an application that is due as much to Andrzej Pelc (see [7]) as to the present author. It answers the following question that we asked in [9, Problem R, p. 51]: Suppose we have countably many $\omega_{1}$-complete uniform ideals on the real line $\mathscr{R}$. Can we then find a subset $X$ of $\mathscr{R}$ such that neither $X$ nor $\mathscr{R}-X$ belongs to any of the ideals in our collection? An affirmative answer to this can be derived as follows. By Theorem 5.4.2 of [9], it suffices to show that if $I$ is an $\omega_{1}$-complete uniform ideal on $\mathscr{R}$ then $\mathscr{P}(\mathscr{R}) / I$ has no countable dense set (in the forcing theoretic sense). Assume we have such an $I$ and $\left\{X_{n}: n \in\right.$ $\omega\} \subseteq I^{+}$. To each distinct $[Y] \in \mathscr{P}(\mathscr{R}) / I$ we associate $\left\{n \in \omega:\left[X_{n}\right] \leq[Y]\right\}$. Since $|\mathscr{P}(\mathscr{R}) / I|>2^{\aleph_{0}}$ by Theorem $2.3\left(\right.$ ii), we get $\left[Y_{1}\right],\left[Y_{2}\right] \in \mathscr{P}(\mathscr{R}) / I$ with $\left[Y_{1}\right]=\left[Y_{2}\right]$ and $\left[X_{n}\right] \leq\left[Y_{1}\right]$ iff $\left[X_{n}\right] \leq\left[Y_{2}\right]$ for every $n \in \omega$. But this shows that $\left\{X_{n}: n \in \omega\right\}$ is not a dense set since either $Y_{1}-Y_{2} \in I^{+}$or $Y_{2}-Y_{1} \in I^{+}$and neither contains $X_{n}$ (modulo $I$ ) for any $n \in \omega$.

A PPLICATION 2. Theorem 2.3(ii) also yields yet another proof that not every set of reals is Lebesgue measurable. To see this, assume the contrary and let $I=\{X \subseteq \mathscr{R}: \mu(X)=0\}$. Then $I$ is clearly $\omega_{1}$-complete and it is uniform since every set of reals of cardinality less than the continuum has outer measure zero. Theorem 2.3(ii) now implies that $|\mathscr{P}(\mathscr{R}) / I|>2^{\aleph_{0}}$, and this contradicts the wellknown fact that every measurable set differs from a Borel set by a set of measure zero (since there are only $2^{\aleph_{0}}$ Borel sets).

A PPLICATION 3. We showed above that a consequence of Theorem 2.3 is the fact that no $\omega_{1}$-complete uniform ideal $I$ on $\mathscr{R}$ can yield a Boolean algebra $\mathscr{P}(\mathscr{R}) / I$ 
with a countable dense set. A natural question (again related to versions of Ulam's problem in [9 and 10]) is whether a dense set of size $\aleph_{1}$ is possible. Woodin [11] has shown that relative to large cardinals an affirmative answer is consistent. In his model $\mathrm{CH}$ holds. As a final application of Theorem 2.2 we consider the question of whether $\mathrm{CH}$ is necessary and prove the following: if $\mathscr{R}$ carries an $\omega_{1}$ complete uniform ideal $I$ so that $\mathscr{P}(\mathscr{R}) / I$ has a dense set of size $\aleph_{1}$ then either $2^{\aleph_{0}}=\aleph_{1}$ or $2^{\aleph_{0}}>\aleph_{\omega_{1}}$. To see this we argue as follows. Suppose $I$ is an $\omega_{1}$ complete, uniform ideal on $\mathscr{R}$ and $D$ is a dense set of size $\aleph_{1}$ for $\mathscr{P}(\mathscr{R}) / I$. Define $\phi: \mathscr{P}(\mathscr{R}) / I \rightarrow \mathscr{P}(D)$ by $\phi([A])=\{d \in D: d \leq[A]\}$. It is easy to see that $\phi$ is one-to-one and so $|\mathscr{P}(\mathscr{R}) / I| \leq 2^{\aleph_{1}}$. On the other hand, Theorem 2.2(ii) guarantees that $|\mathscr{P}(\mathscr{R}) / I|>2^{\aleph_{0}}$, and so $2^{\aleph_{0}}<2^{\aleph_{1}}$. Now, if $\kappa$ is the exact completeness of $I$ it is easy to see that $I$ projects to a $\kappa$-complete ideal on $\kappa$, and since $I$ has a dense set of size of $\omega_{1}$ it is clearly $\omega_{2}$-saturated. Thus, if $2^{\aleph_{0}}<\aleph_{\omega_{1}}$ we must have $\kappa=\omega_{1}$ and so we arrive at a situation where we have an $\omega_{2}$-saturated $\omega_{1}$-complete ideal on $\omega_{1}$ with $2^{\aleph_{0}}<2^{\aleph_{1}}$. A well-known result of Jech-Prikry [4, p. 3] now yields $2^{\aleph_{0}}=\aleph_{1}$ as desired.

\section{REFERENCES}

1. C. C. Chang and H. J. Keisler, Model theory, North-Holland, Amsterdam, 1973.

2. W. W. Comfort and A. W. Hager, Cardinality of $\lambda$-complete Boolean algebras, Pacific J. Math. 40 (1972), 541-545.

3. T. Frayne, A. C. Morel and D. S. Scott, Reduced direct products, Fund. Math. 51 (1962), 195-228.

4. T. Jech and K. Prikry, Ideals over uncountable sets: applications of almost disjoint functions and generic ultrapowers, Mem. Amer. Math. Soc. no. 214 (1979).

5. H. J. Keisler, On cardinalities of ultrapowers, Bull. Amer. Math. Soc. 70 (1964), 644-647.

6. J. D. Monk and P. R. Sparks, Counting Boolean algebras, Notices Amer. Math. Soc. 18 (1971), 551.

7. A. Pelc, Ideals on the real line and Ulam's problem, Fund. Math. 109 (1980).

8. K. Prikry, Ideals and powers of cardinals, Bull. Amer. Math. Soc. 81 (1975), 907-909.

9. A. D. Taylor, On saturated sets of ideals and Ulam's problem, Fund. Math. 109 (1980), 37-53.

10. __ Regularity properties of ideals and ultrafilters, Ann. Math Logic 16 (1979), 33-55.

11. H. Woodin, Some consistency results in ZF using AD, Cabal Seminar, Lecture Notes in Math., vol. 1019, Springer, pp. 172-199.

Department of Mathematics, Union College, Schenectady, New York 12308 